\title{
Investigación científica, piedra angular en la generación del conocimiento
}

CIENCIA Y TECNOLOGÍA(CT) Recientemente se publicó el Ranking lberoamericano SIR 2010 un índice que se genera a partir del número de documentos científicos publicados, la capacidad de las universidades para realizar trabajos en colaboración con otros países y la visibilidad de la institución. El Ranking posiciona a la UNAH en el puesto 364 de 607 universidades de lberoamérica y en el puesto 9 entre las 33 universidades de Centroamérica en investigación científica.

Para analizar los resultados obtenidos por la UNAH y cómo superarlo, CIENCIA Y TECNOLOGÍA entrevistó a tres connotados docentes investigadores de diferentes áreas del conocimiento de la UNAH: al sociólogo Ramón Ulises Salgado Peña; a la científica en Ciencias Espaciales, María Cristina Pineda y a el científico en neurociencias, doctor Marco Tulio Medina Hernández.

A continuación la entrevista con Ramón Ulises Salgado Peña, docente en el área de ciencias sociales de la UNAH, cuyas investigaciones se han centrado en el agro y la educación.

\section{$\mathrm{CT} /$ ¿Cómo valora la importancia de los rankings internacionales?}

RUSPI Estos rankings que están apareciendo sobre las universidades son una herramienta muy útil que permite contar con el criterio de una visión externa debido a que son elaborados a nivel internacional. Son un punto de referencia importante. Es un honor y a la vez un desafío que obliga a la UNAH a mejorar suposición. Deben tomarse como un dato positivo.

\section{CT/ ¿Cómo mejorar esta posición a largo plazo?}

RUSP/ La mejora tiene que ver con decisiones internas que se están haciendo a lo interior de la Universidad. La Dirección de Investigación Científica está apostando al desarrollo académico y de manera particular al desarrollo de la investigación. Lo que se busca es profundizar en las diferentes áreas como la capacitación de los profesionales, la internacionalización de la UNAH que significa vincularse con profesores internacionales de universidades de prestigio. Los concursos de investigación que se están haciendo y el apoyo a los profesionales universitarios para investigar son un buen incentivo. Falta desarrollar fuertemente la incorporación de los estudiantes de pregrado y postgrado. Cuando uno ve los rankings en donde están universidades como Harvard se siente el peso que le dan a la participación estudiantil en la investigación.

CT/ ¿Se cuenta con el talento humano necesario para formar núcleos de investigadores? 
RUSPI Diría que la UNAH, al igual que el resto de las universidades en el país, no está conceptualizada como una institución de investigación. Las principales universidades que aparecen en los rankings internacionales son de investigación, pero se puede hacer un esfuerzo futuro para darle más apertura al campo de la investigación. Los talentos humanos se tienen, pero se requerirá de recursos financieros más allá de la UNAH, yo diría del Estado para la adquisición del equipo especializado que demandan los investigadores. Existe el talento humano necesario, pero hay que ampliar la base .

\section{CT/ ¿Cómo investigar, si se cuenta con un presupuesto limitado?}

RUSP/ Las universidades que están orientadas a la investigación combinan los fondos estatales con recursos de la cooperación internacional y con los de empresas y/o organismos internacionales que colocan fondos concursables para el campo de la investigación. Entonces, si se tiene los talentos humanos y una política de captación de recursos a nivel internacional, lo que se debe hacer es gestionar en sus diferentes campos la obtención de esos fondos. Ese es uno de los desafíos que tiene la UNAH.

\section{$\mathrm{CT} /$ ¿Por qué la investigación de la UNAH se concentra en las ciencias médicas?}

RUSPI Bueno, son las áreas que más continuidad han tenido. En la década de los $70 \mathrm{y}$ los 80 del siglo pasado hubo mucha investigación en el campo de las ciencias sociales: Sociología, Historia y Trabajo Social, entre otras carreras. Lo que estamos observando es un cambio generacional. Están cambiando las generaciones de docentes y lamentablemente no se ha contado con un sistema de relevo formado al más alto nivel. Ahora hay más apertura para que resurgan los estudios en los campos de las humanidades en general y de las ciencias sociales en particular. Las ciencias médicas, por mucho tiempo, han tenido una tradición de trabajo, con producción científica, publicación en revistas especializadas y profesores internacionales.

\section{$\mathrm{CT} /$ ¿Cómo consolidar este proceso, que otras administraciones no lo retresen?}

RUSP/ Creo que este proceso es irreversible porque va acompañado de la aprobación de mecanismos legales, además se está consolidando toda una masa crítica de investigadores que vuelve difícil el retroceso. Los frutos se verán en los próximos años.

\section{CT/ ¿Cómo motivar al docente para que además de educar, también investigue?}

RUSP/ La UNAH está obligada a preparar las mejores condiciones, pero investigar es algo personal. Le debe gustar al investigador, no se puede obligar a nadie. Pero sí se pueden crear las condiciones que motiven a esos talentos individuales, para luego agruparlos en equipos de investigación que den respuesta a los retos que plantea el país. 


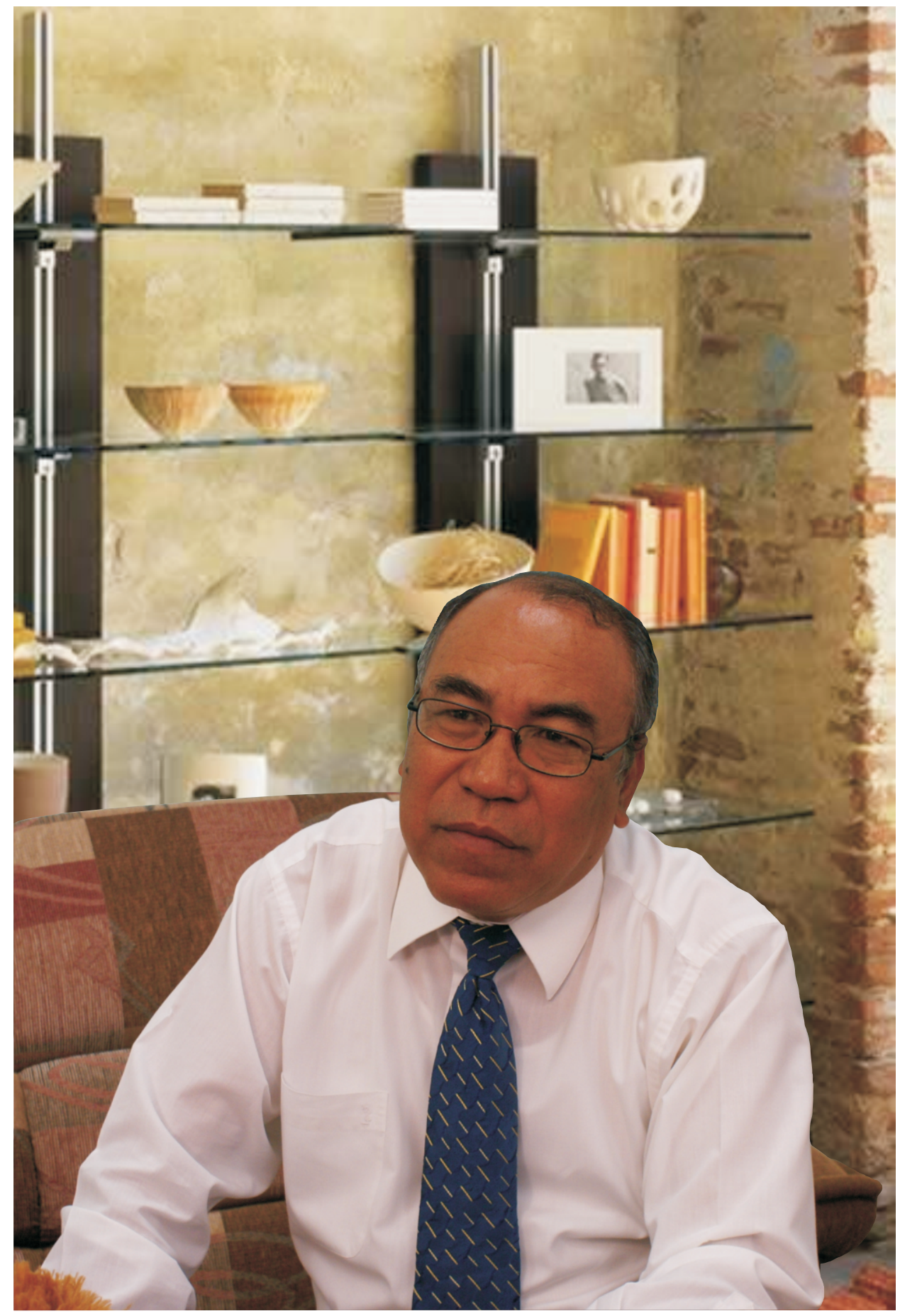

Sociólogo Ramón Salgado Peña

Dirección de Investigación Científica $\longdiv { 1 3 }$ 


\section{CIENCIA Y TECNOLOGÍA (CT) Entrevista con la doctora María Cristina Pineda, decana de la Facultad de Ciencias Espaciales. Esta hondureña es estudiosa de los campos de la Astronomía y Astrofísica, Ciencia y Tecnología de la Información Geográfica y Arqueoastronomía.}

\section{CT/ ¿Desde la Facultad de Ciencias Espaciales cómo se incentiva el proceso de Investigación Científica?}

MCP / Cuando uno desarrolla cierto tipo de actividad desde un campo especializado del conocimiento, el investigador está dando contribuciones para que la institución universitaria sea categorizada dentro del ranking de universidades. En la facultad de Ciencia Espaciales, como una política institucional, todos los profesores participan en el desarrollo de la investigación científica, con lo que se está garantizando aportes al conocimiento bastantes significativos. La producción de la facultad de Ciencias Espaciales se mide desde tres aspectos: la publicación continúa de sus resultados, que ha dado lugar a nuestra revista especializada "Ciencias Espaciales" que comprende los campos de la Astronomía y Astrofísica, las Ciencias y Tecnologías de la Información Geográfica y Arqueoastronomía. Un segundo aspecto es la presentación continua de nuestros resultados a la comunidad científica nacional e internacional. Esto quiere decir que estamos participando en congresos nacionales e internacionales especializados. En nuestra última participación en el congreso especializado alcanzamos una marca muy importante porque tuvimos la presencia de científicos de varias universidades de España, Argentina, Nicaragua, Panamá, Uruguay, México, República Dominicana y Honduras, que han venido a ver cómo está el estado del conocimiento en la UNAH. Inmediatamente que nosotros terminamos el congreso, una universidad mexicana pidió ser la sede del nuevo conclave científico. $O$ sea que hemos dado pie a un campo especializado importante. Un tercer aspecto es la divulgación de nuestras labor a través de una página web donde se ubican los resultados de las investigaciones. Cualquier persona que desee establecer contactos con grupos de investigadores lo puede hacer a través de nuestro sitio. Ciencias Espaciales ha contribuido a que dentro de las instituciones privadas de educación superior, la UNAH sea la líder en el ranking de las universidades internacionales.

\section{CT/ ¿Por qué es importante la vinculación de la UNAH con otras universidades del mundo?}

MCPI El conocimiento es patrimonio de la universidad. Cada persona, en distintos lugares, tiene diferentes aproximaciones, formas de abordar los problemas. Al fin y al cabo, el desarrollo de la ciencia se hace a través de la solución de problemas, pero cuando uno tiene diferentes perspectivas, lados, lógicamente hay garantías de obtener mejores aciertos y mayores avances. Nosotros, toda nuestra actividad la hacemos con grupos de investigadores de universidades internacionales, y es que al vivir en la era del conocimiento, es la única forma de hacerlo. Trabajar aisladamente, con la idea 
fantástica del científico solitario que trabaja en su laboratorio, eso quedó para la historia. Hoy la persona que no se relaciona, que no publica, que no está en contacto con la comunidad científica internacional, perece.

\section{CT/ ¿Cuál es el vínculo de la Facultad de Ciencia Espaciales con la universidad de Alcalá, cuáles son los resultados?}

MCP/ Trabajamos dentro del espacio que la cooperación le llama "Cooperación para el desarrollo". No basta trabajar con alguien más, no basta cooperar, tiene que ser internacional. Eso quiere decir que formamos parte de la gran familia de profesionales del campo que hemos venido formando las dos universidades. Y esto nos ha dado la gran ventaja de trabajar con diferentes universidades del mundo, además de aprovechar lo propio que tiene la universidad de Alcalá. En ese marco de la cooperación internacional para el desarrollo, como institución, tenemos el compromiso de que a la vez que desarrollamos nuestra actividad, debemos ir desarrollando el campo para beneficio y el engrandecimiento del país, y ese es un reto grande, sobre todo porque tienen estándares muy altos. Se nos exige publicar y organizar nuestros laboratorios de determinada manera. Se nos exige formar a nuestros estudiantes con determinado tipo de conocimientos, habilidades y aptitudes de continua investigación, vinculación y publicación, dando como resultado profesionales de éxito.

\section{$\mathrm{CT} /$ ¿Cuáles son los resultados del Congreso de Ordenamiento Territorial?}

MCP/ La oportunidad de poder presentar en la sociedad hondureña y en la región centroamericana cómo las ciencias de la tecnología de la información geográfica han avanzado. Se discutió lo último del conocimiento, y esto es importante porque es un termómetro para que las instituciones, públicas y privadas, se den cuenta cómo es que están trabajando. Algo muy importante es el hecho de que el espacio sirvió para que la academia, por medio de la Facultad de Ciencias Espaciales, el gobierno, a través de la Dirección General de Catastro y Geografía y la Dirección de Ordenamiento Territorial y la empresa privada, con la participación de Ingeniería Gerencial se integraran por el desarrollo del país. Ese es quizá uno de los logros más importantes. Esto lo están viendo nuestros estudiantes de pregrado y post grados, o sea que se están haciendo marcas que las nuevas promociones tienen que superar.

\section{$\mathrm{CT} /$ ¿Qué retos tiene la universidad frente a esta temática?}

MCP/ La universidad dio un paso importante al crear la Facultad de Ciencias Espaciales, pero hoy toda la comunidad universitaria tiene que enterarse de cuáles son los campos que estamos desarrollando. Por el lado de nosotros, insertarnos en esos proyectos interdisciplinarios y multidisciplinarios para demostrarle a la sociedad que nosotros sí llevamos el liderazgo en este tipo de cambios. 


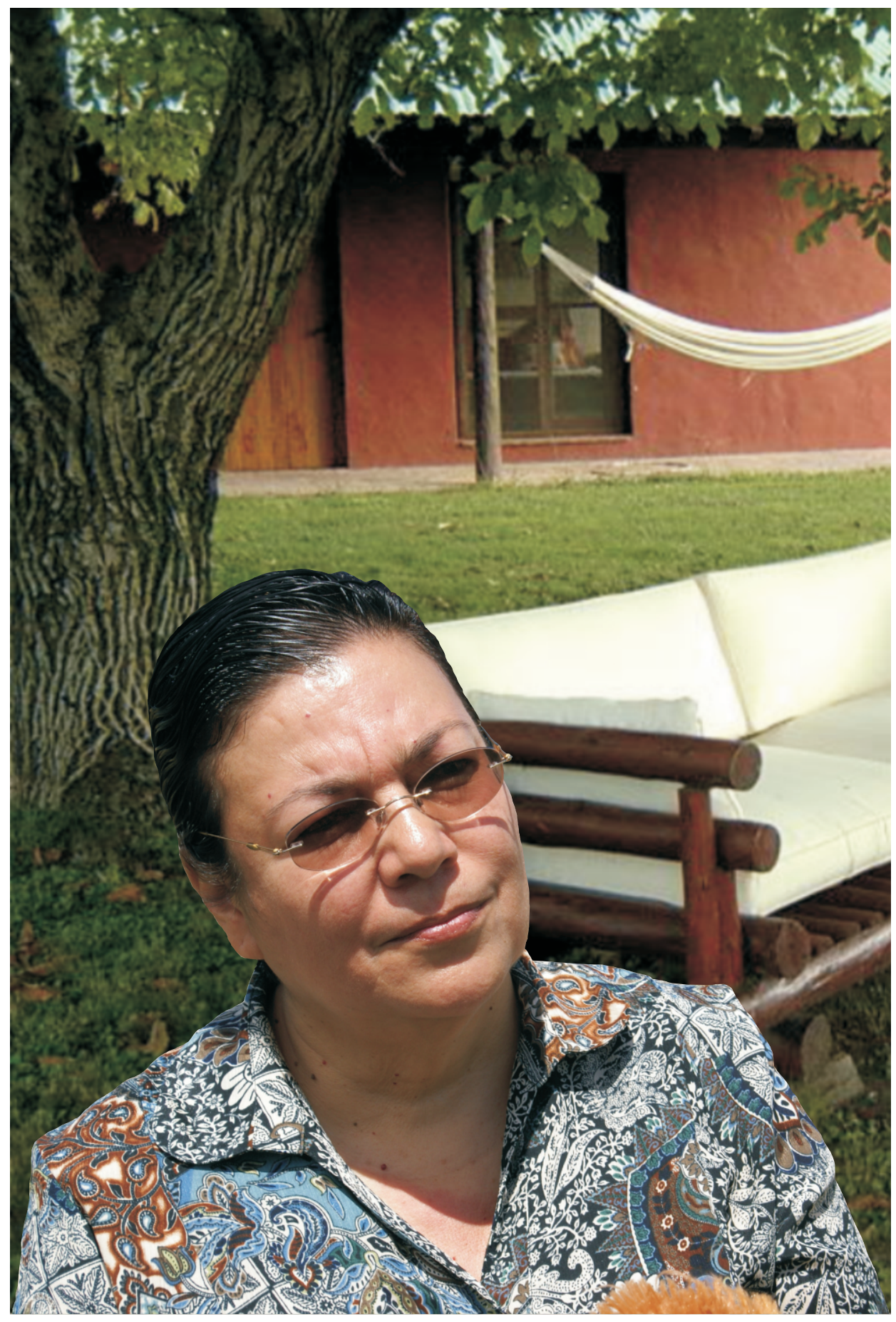

Doctora María Cristina Pineda

16 Dirección de Investigación Científica 
Entrevista con el doctor Marco Tulio Medina Hernández, decano de la facultad de Ciencias Médicas de la UNAH. Su temática de investigación ha girado en torno a la neurología, la genética de la epilepsia, enfermedades vasculares cerebrales y enfermedades degenerativas, entre otras.

\section{$\mathrm{CT} /$ ¿Cómo valora los resultados de los rankings internacionales en investigación?}

MTMH/ Permiten conocer la posición que tiene una universidad dentro del contexto de universidades, en este caso de Latinoamérica. Son importantes porque definen con criterios sólidos y científicos el nivel de una universidad. Para la UNAH, el estar ubicada dentro del grupo intermedio de la medición es un indicador de que se está frente a un gran reto por convertirnos en una de las mejores universidades de América Latina. Tenemos la capacidad para ubicarnos entre las primeras tres posiciones de América Central, superando así la posición 9 de entre las 33 en que nos encontramos. Para superar la posición obtenida en el actual Ranking SIR 2010, se debe tener una posición muy orientada hacia la investigación.

\section{CT/ ¿Está progresado en la UNAH el proceso de investigación. Cómo acelerarlo?}

MTMH/Existen varios grupos que están produciendo ciencia y que se publica en revistas indexadas de impacto mundial. Dentro de estos grupos está el de Neurología, que se ubica en el primer lugar; Microbiología, Ciencias Espaciales y Biología. Estos son los cuatro grupos que más producción han tenido en la UNAH. Además, hay grupos que están creciendo fuertemente como el de Ciencias Sociales, provocando un aumento en la producción de la investigación científica en los últimos años. Sin embargo, la velocidad de ese incremento debe ser mejorada y para que mejore se necesitan más núcleos de investigadores que optimicen el sistema a nivel universitario. Se debe fomentar que los investigadores maduros trabajen con grupos de jóvenes para dar paso a la formación de núcleos de investigación. A los investigadores se les debe apoyar con becas especiales. Otra forma de incentivarlos es difundiendo entre la comunidad universitaria lo qué se está haciendo en el campo de la investigación. Estos son incentivos morales que promueven un alto estándar entre los investigadores. Esto sirve de ejemplo a los jóvenes, que más tarde los motiva a formar parte de los grupos de investigadores. Indudablemente, la creación de la infraestructura de investigación es fundamental, así como el fomento del trabajo multidisciplinario al seno de la universidad. Se debe lograr que el producto de las investigaciones sean insumos para el proceso de educación.

\section{CT/ ¿Qué significa para Honduras el aumento de la producción científica?}

MTMH/ La UNAH debe dejar de ser una institución repetidora del conocimiento extranjero, para transformarse en una universidad innovadora que produce ciencia. Esto quiere decir que la línea de investigación debe ser el eje educativo. En otras 
universidades, los datos científicos nuevos son transmitidos en el seno de una universidad, manteniendo un proceso constante de innovación en la educación.

\section{CT/ ¿Cómo repercute en el país el depender del conocimiento importado?}

MTMH/Esto es producto de la toma de decisiones dentro del sistema estatal nacional, de cuánto se está invirtiendo en ciencia y tecnología en el país. Para el caso, en los últimos 50 años algunos países de Asia han invertido en la investigación de las universidades, lo que provocó que estas sociedades asiáticas dieran un salto cualitativo que las ha equiparado al nivel de sociedades europeas y estadounidenses. Es así, que uno de los factores determinantes es cuánto se invierte en un área prioritaria como la ciencia y tecnología. Cuando se invierte en ciencia y tecnología se generan usualmente patentes, nuevo conocimiento, nuevas estrategias para generar más producción económica con solidez.

\section{$\mathrm{CT} /$ ¿Qué se hace desde Ciencias Médicas para incentivar este proceso?}

MTMH/ La facultad tiene 128 años de fundación. Durante este tiempo siempre se ha inclinado por la investigación, dando como resultado la generación de reconocidos científicos hondureños de alta valía como Hernán Corrales Padilla, Pablo J. Cámbar y Salvador Moncada, que estuvo trabajando en nuestra facultad. Tenemos dos revistas científicas, la de postgrado y la de pregrado, existe una unidad de Investigación Científica con más de 15 años de trayectoria. Los médicos en servicio social para graduarse tienen que hacer un trabajo grupal de investigación y se cuenta con grupos de investigadores que están siendo promovidos en el área de salud pública. Existe una página web de la Biblioteca Médica Nacional, adscrita a la Organización Panamericana de la Salud. Pese a todo, sabemos que hay muchos retos, que la producción científica tiene que ser aumentada en forma significativa para alcanzar mejores niveles.

\section{$\mathrm{CT} /$ ¿Qué tipo de colaboración externa se recibe para potenciar la investigación?}

MTMH/Se han suscrito convenios con universidades norteamericanas y de Europa y se ha creado una red de investigadores con hondureños con excelencia que viven fuera de Honduras y que bajo la estrategia nacional "Honduras Global" apoyan a la facultad. Por otro lado, Medicina es la única facultad que tiene un comité de bioética para revisar los estándares éticos.

\section{$\mathrm{CT} /$ ¿Por qué algunos investigadores emigran de Honduras?}

MTMH/ Invertir en un investigador es promover su trabajo, es proveerlo de los insumos apropiados y del tiempo necesario. En la UNAH se deben crear las condiciones para apoyarlos decididamente y evitar su salida del país o su retiro de la actividad investigativa. 


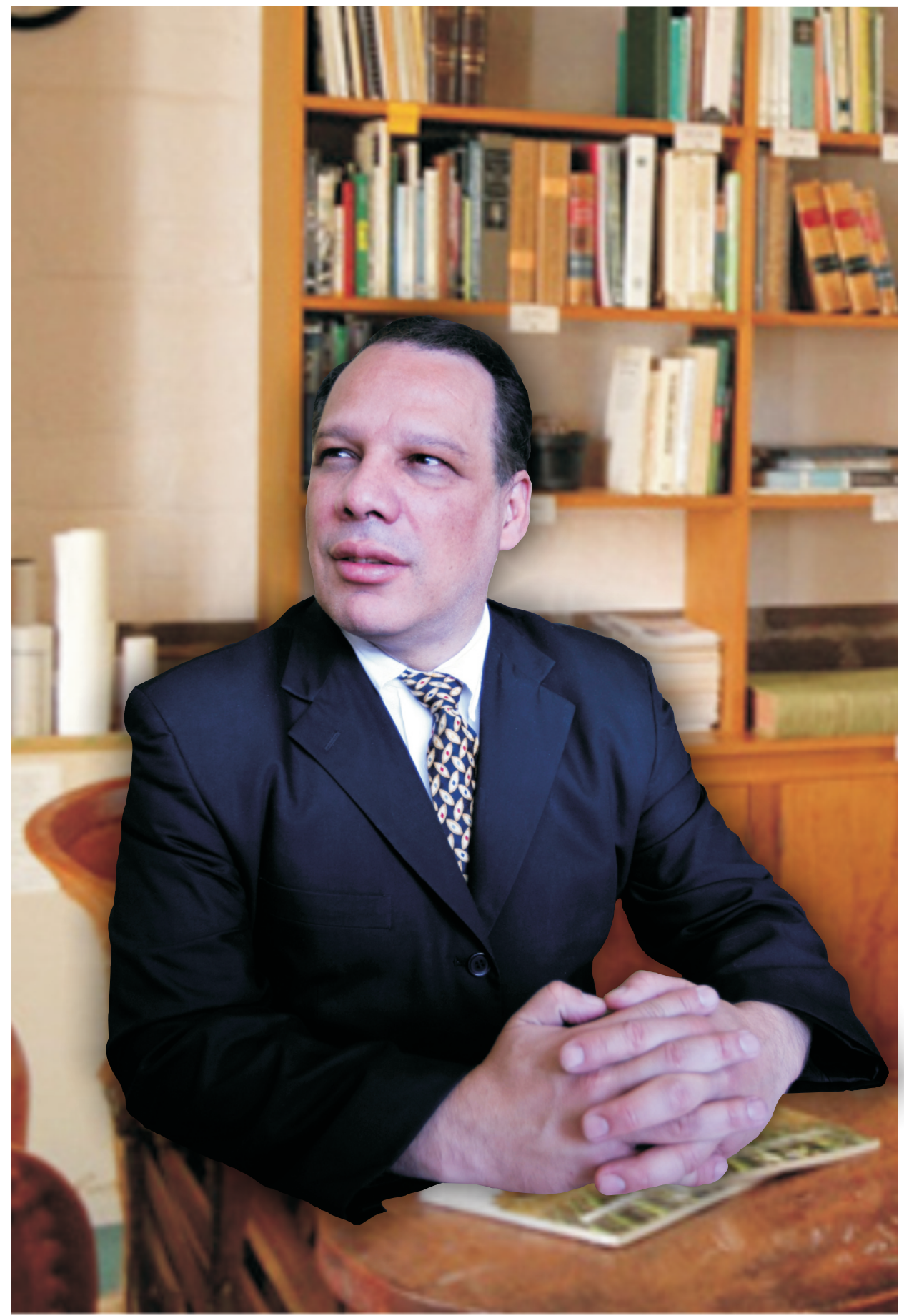

Doctor Marco Tulio Medina Hernández

Dirección de Investigación Científica $\longdiv { 1 9 }$ 